\title{
Hubble Space Telescope Observations of Millisecond Pulsar Companions: Constraints on Evolution
}

\author{
S. C. Lundgren and R. S. Foster \\ Remote Sensing Division, Code 7213, Naval Research Laboratory, \\ Washington, DC 20375 \\ F. Camilo \\ The University of Manchester, NRAL, Jodrell Bank, Macclesfield, \\ Cheshire SK11 9DL, UK
}

\begin{abstract}
In observations of six binary millisecond pulsars with the Hubble Space Telescope, we have discovered white dwarf companions to PSRs J0034-0534, J1022+1001, J1713+0747, and J2019+2425 and improved photometry on PSRs J1640+2224 and J2145-0750. Three of the white dwarfs are among the coolest and oldest known. We have determined that the masses for the helium companions are consistent with the expectation based on the core mass of a progenitor that filled its Roche lobe. The cooling times for many of the white dwarfs are much less than the characteristic spin-down times, implying that the spin period at the end of the accretion stage was close to the current period. The initial spin periods calculated are used to place limits on the accretion rate at the end of the low-mass X-ray binary phase. The accretion rates are found to be over an order of magnitude less than the Eddington rate.
\end{abstract}

\section{Introduction and Observations}

Significant progress has been made recently in identifying companions to millisecond pulsars (MSPs). White dwarf companions have been detected optically for pulsars J0437-4715, J1012+5307, J1640+2224, and J2145-0750 (Bell, Bailes, \& Bessell 1993; Danziger, Baade, \& Della Valle 1993; Lorimer et al. 1995; Lundgren et al. 1996; Bell et al. 1995). We report on new Hubble Space Telescope (HST) ${ }^{1}$ optical observations of companions to six binary millisecond pulsars. From optical studies, the temperature, radius, and mass of the companion can be determined. The current characteristics of the companion yield insight into the past evolution of the binary system. A large sample of optically detected companions tests several aspects of the standard model for binary pulsar evolution: the initial spin period/magnetic field relation from the accretion spin-up process, the orbital period/companion mass relation from Roche lobe

\footnotetext{
${ }^{1}$ Based on observations with the NASA/ESA Hubble Space Telescope, obtained at the Space Telescope Science Institute
} 
Table 1. Pulsar, Companion, and Orbital Parameters

\begin{tabular}{crcrrrrc}
\hline Pulsar & $\begin{array}{r}b \\
(\mathrm{deg})\end{array}$ & $\begin{array}{c}d \\
(\mathrm{kpc})\end{array}$ & $\begin{array}{r}\tau_{s} \\
\left(10^{9} \mathrm{yr}\right)\end{array}$ & $\begin{array}{r}P \\
(\mathrm{~ms})\end{array}$ & $\begin{array}{r}P_{b} \\
(\text { days })\end{array}$ & $\begin{array}{r}m_{c, \text { mfn }} \\
\left(M_{\odot}\right)\end{array}$ & $\begin{array}{c}m_{c, \text { cor }} \\
\left(M_{\odot}\right)\end{array}$ \\
\hline $\mathrm{J} 0034-0534$ & -68.1 & 1.0 & 5.9 & 1.88 & 1.6 & 0.17 & 0.16 \\
$\mathrm{~J} 1022+1001$ & 51.1 & 0.6 & 6.2 & 16.45 & 7.8 & 0.87 & - \\
$\mathrm{J} 1640+2224$ & 38.3 & 1.2 & $>20$ & 3.16 & 175 & 0.30 & 0.31 \\
$\mathrm{~J} 1713+0747$ & 25.2 & 1.1 & 9.6 & 4.57 & 68 & 0.33 & 0.27 \\
$\mathrm{~J} 2019+2425$ & -6.6 & 0.9 & 24 & 3.93 & 77 & 0.37 & 0.28 \\
$\mathrm{~J} 2145-0750$ & -42.1 & 0.5 & 9.0 & 16.05 & 6.8 & 0.51 & - \\
\hline
\end{tabular}

overflow driven by nuclear evolution of the white dwarf progenitor (Rappaport et al. 1995, hereafter RPJ95), and the ages from white dwarf cooling models.

In Table 1 the properties of the selected MSPs are listed (Taylor et al. 1995). The distances $(d)$ are estimated with $25 \%$ uncertainty from the dispersion of the radio signal using the Taylor \& Cordes (1993) model for the density of the interstellar plasma. For some of the pulsars a better distance will eventually be determined from timing parallax (e.g. Camilo, Foster, \& Wolszczan 1994) or from the apparent change in orbital period due to the doppler effect from proper motion (Bell \& Bailes 1996). The spin-down times $\left(\tau_{s}\right)$ have been corrected for proper motion effects. The companion mass is estimated both from the median mass allowed by the mass function $\left(m_{c, \mathrm{mfn}}\right)$ and from the RPJ95 model for the core mass $\left(m_{c, \text { cor }}\right)$ of a Roche-lobe filling giant star in the observed orbit.

The sources were imaged with the Planetary Camera (PC2) on HST. Integration times used for the $439 \mathrm{~nm} \mathrm{~B}, 555 \mathrm{~nm} \mathrm{~V}$, and $814 \mathrm{~nm}$ I filters were $2100 \mathrm{~s}$, $1000 \mathrm{~s}$, and $800 \mathrm{~s}$ respectively. The positions of our sources have uncertainties of $1^{\prime \prime}$ due to the uncertainty in the offset between the PC2 and the Fine Guidance Sensors. We used the STSDAS and PHOT packages within IRAF for data reduction and calibration, following the steps in the HST Photometry Handbooks (Holtzman et al. 1995a; Whitmore 1995). We used 4 pixel radius apertures and applied the aperture corrections of Holtzman et al. (1995). Finally, we converted from the HST filter system to the standard Kron-Cousins BVRI-system.

\section{Results and Conclusions}

All six pulsar companions were detected in our HST observations. In Table 2 we have compiled the results. The absolute luminosity and $V-I$ and $B-V$ colors take into account the distance modulus $(m-M)$ from the pulsar dispersion distance and the reddening $\left(E_{B-V}\right)$ and extinction from Burstein and Heiles (1982). In all cases, the companion must be a white dwarf, because a main sequence star at the observed temperature and distance would be about four magnitudes brighter than the observed star. The temperature calibration of the colors and the bolometric correction $\left(m_{b}-m_{I}\right)$ have been determined by Monet et al. (1992) and Bergeron, Saumon \& Wesemael (1995).

The mass $\left(m_{2}\right)$ of each white dwarf has been inferred from the photometry. For assumed black body emission, the luminosity, temperature, and distance 
Table 2. Photometry*

\begin{tabular}{lrrrrrr}
\hline Parameter & J0034-05 & J1022+10 & J1640+22 & J1713+07 & J2019+24 & J2145-07 \\
\hline pos'n & & & & & & \\
offset $\left(^{\prime \prime}\right)$ & 0.6 & 1.0 & 0.8 & 0.5 & 0.3 & 0.9 \\
$m_{B}$ & - & - & - & $>27.1$ & - & $23.89(11)$ \\
$m_{V}$ & $>26.8$ & $23.09(4)$ & $26.0(3)$ & $26.0(2)$ & $26.4(4)$ & $23.70(10)$ \\
$m_{I}$ & $24.8(3)$ & $22.665(7)$ & $24.6(2)$ & $24.1(1)$ & $25.0(3)$ & $22.97(7)$ \\
$B-V$ & - & - & - & $>1.1$ & - & $0.18(14)$ \\
$V-I$ & $>2.0$ & $0.43(4)$ & $1.4(4)$ & $1.9(2)$ & $<1.1$ & $0.73(10)$ \\
$m-M$ & $10.0(5)$ & $8.9(5)$ & $10.4(5)$ & $10.2(5)$ & $9.8(5)$ & $8.5(5)$ \\
$E_{B-V}$ & $0.00(2)$ & $0.00(2)$ & $0.05(2)$ & $0.08(2)$ & $>0.2$ & $0.03(2)$ \\
\hline $\mathrm{T}(\mathrm{K})$ & $<3800$ & $6925(200)$ & $4200(300)$ & $3700(100)$ & $>4500$ & $5800(300)$ \\
$\log \left(L / L_{\odot}\right)$ & $-4.2(2)$ & $-3.8(2)$ & $-3.8(2)$ & $-3.8(2)$ & $>-4.4$ & $-4.1(2)$ \\
$m_{2} / M_{\odot}$ & $0.23(20)$ & $0.83(25)$ & $0.25(10)$ & $<0.32$ & $0.6(3)$ & $0.87(25)$ \\
$t_{\text {cool }}(\mathrm{Gyr})$ & $>4.5$ & $3.7(9)$ & $7(2)$ & $8(2)$ & $<8$ & $5.5(7)$ \\
$P_{i}(\mathrm{~ms})$ & - & $10(2)$ & $>2.5(2)$ & - & $>3.2$ & $10(2)$ \\
$\dot{M} / \dot{M}_{\text {edd }}$ & - & $0.007(2)$ & $0.005(1)$ & - & $<0.002$ & $0.010(3)$ \\
\hline
\end{tabular}

* The numbers in parentheses represent the uncertainty in the last digits quoted.

imply a radius for the white dwarf, $\log \left(R / R_{\odot}\right)=1 / 5\left(42.31-m_{b}-10 \log T_{e}\right)$. The radius in turn implies a mass through the well known white dwarf mass radius relation (Hamada \& Salpeter 1961). The largest uncertainty in the mass estimate comes from the $25 \%$ distance uncertainty. In most of the systems, the mass inferred from the optical observations agrees with the mass determined from the RPJ95 model or from the mass function, for distances consistent with the dispersion distance. Only PSR J1713+0747 requires a distance substantially different from the dispersion distance.

The age of the white dwarfs were determined from white dwarf cooling models. For the 3 more massive white dwarfs we use the carbon-oxygen models of Wood (1992). Cooling times for the less massive helium dwarfs have been calculated by scaling the luminosity in Wood's models by $m_{2}^{5 / 7} / A$ where $A$ is the atomic number of the stellar matter. For all but PSRs J0034-0534 and $\mathrm{J} 1713+0747$, the cooling time is much less than the spin-down time of the pulsar, indicating that the initial spin period of the pulsar was close to the current period. The body of evidence from white dwarf cooling now indicates that MSP spin-down times should not be used as ages for the systems, but rather only as upper limits to the age. However, we reject the earlier hypothesis that many MSPs may be an order of magnitude or more younger than the age of the Galaxy, as suggested in earlier work (Lorimer et al. 1995), based on finding one white dwarf a factor of 20 younger than its spin-down time. Quite to the contrary, we find that the distribution of MSP ages is consistent with a uniform distribution stretching nearly to the age of the Galaxy ( $10 \mathrm{Gyr})$.

Assuming a magnetic dipole spin-down rate, we have estimated the initial spin periods for the pulsars with cooling times much smaller than spin-down times. Bhattacharya and van den Heuvel (1991) reviewed a model relating the initial spin period $\left(P_{i}\right)$ of the pulsar to the final accretion rate $\left(\dot{M} / \dot{M}_{e d d}\right)$ in 
the low-mass X-ray binary phase, where $\dot{M}_{e d d}$ is the Eddington accretion rate. We have found that $\dot{M}<<\dot{M}_{\text {edd }}$ for all of these pulsars. Apparently, MSPs do not always reach the so-called "spin-up line"-the line on the magnetic field vs. period diagram defined by the equilibrium period of a pulsar accreting at the Eddington rate. In the case of the intermediate mass systems, this is not surprising. The common envelope phase was too short for the pulsar to reach the equilibrium period. Instead, the initial period may have resulted from sub-Eddington accretion from the stellar wind of the giant star before the asymptotic giant phase (van den Heuvel 1994). However, even in systems like PSR J1640+2224 with long-term stable mass transfer, apparently the accretion rate was not sufficient for the pulsar to reach the "spin-up line".

SCL is supported by a fellowship through the National Research Council. Primary support was provided by NASA through a grant from the Space Telescope Science Institute, which is operated by the Association of Universities for Research in Astronomy, Inc. under NASA contract NAS5-26555. Basic research in precision pulsar astrophysics at the Naval Research Laboratory is supported by the Office of Naval Research. FC gratefully acknowledges a fellowship under the auspices of the European Commission.

\section{References}

Bell, J. F. \& Bailes, M. 1996, Astrophys. J., 456, L33

Bell, J. F., Bailes, M., \& Bessell, M. S. 1993, Nature, 364, 603

Bell, J. F., Kulkarni, S. R., Bailes, M., Leitch, E. M., \& Lyne, A. G. 1995, Astrophys. J., 452, L121

Bergeron, P., Saumon, D., \& Wesemael, F. 1995, Astrophys. J., 443, 764

Bhattacharya, D. \& van den Heuvel, E. P. J. 1991, Phys. Rep., 203, 1

Burstein, D. \& Heiles, C. 1982, Astron. J., 87, 1165

Camilo, F., Foster, R. S., \& Wolszczan, A. 1994, Astrophys. J., 437, L39

Danziger, I. J., Baade, D., \& Della Valle, M. 1993, Astr. Astrophys., 276, 382

Hamada, T. \& Salpeter, E. E. 1961, Astrophys. J., 134, 683

Holtzman, J. A., Burrows, C. J., Casertano, S., Hester, J. J., Trauger, J. T.,

Watson, A. M., \& Worthey, G. 1995a, Publ. Astr. Soc. Pacific, 107, 1065

Holtzman, J. A. et al. 1995b, Publ. Astr. Soc. Pacific, 107, 156

Lorimer, D. R., Lyne, A. G., Festin, L., \& Nicastro, L. 1995, Nature, 376, 393

Lundgren, S. C., Cordes, J. M., Foster, R. S., Wolszczan, A., \& Camilo, F. 1996, Astrophys. J., 458, L33

Monet, D. G., Dahn, C. C., Vrba, F. J., Harris, H. C., Pier, J. R., Luginbuhl,

C. B., \& Ables, H. D. 1992, Astron. J., 103, 638

Rappaport, S., Podsiadlowski, P., Joss, P. C., DiStefano, R., \& Han, Z. 1995, Mon. Not. R. astr. Soc., 273,731

Taylor, J. H. \& Cordes, J. M. 1993, Astrophys. J., 411, 674

Taylor, J. H., Manchester, R. N., Lyne, A. G., \& Camilo, F. 1995, unpublished, anonymous ftp at [pulsar.princeton.edu]:pub/catalog

van den Heuvel, E. P. J. 1994, Astr. Astrophys., 291, L39

Whitmore, B. 1995, in Photometry with the WFPC2, http://www.stsci.edu/

Wood, M. A. 1992, Astrophys. J., 386, 539 